\title{
HAMILTONIAN STATIONARY CONES WITH ISOTROPIC LINKS
}

\author{
JINGYI CHEN AND YU YUAN \\ In memory of Professor Wei-Yue Ding
}

\begin{abstract}
We show that any closed oriented immersed Hamiltonian stationary isotropic surface $\Sigma$ with genus $g_{\Sigma}$ in $S^{5} \subset \mathbb{C}^{3}$ is (1) Legendrian and minimal if $g_{\Sigma}=0$; (2) either Legendrian or with exactly $2 g_{\Sigma}-2$ Legendrian points if $g_{\Sigma} \geq 1$. In general, every compact oriented immersed isotropic submanifold $L^{n-1} \subset S^{2 n-1} \subset \mathbb{C}^{n}$ such that the cone $C\left(L^{n-1}\right)$ is Hamiltonian stationary must be Legendrian and minimal if its first Betti number is zero. Corresponding results for non-orientable links are also provided.
\end{abstract}

\section{INTRODUCTION}

In this note we study the problem of when a Hamiltonian stationary cone $C(L)$ with isotropic link $L$ on $S^{2 n-1}$ in $\mathbb{C}^{n}$ becomes special Lagrangian. A submanifold $M \subset \mathbb{C}^{n}$, not necessarily a Lagrangian submanifold, is Hamiltonian stationary means

$$
\operatorname{div}_{M}(J H)=0,
$$

where $J$ is the complex structure in $\mathbb{C}^{n}$ and $H$ is the mean curvature vector of $M$ in $\mathbb{C}^{n}$. In fact this is the variational equation of volume of $M$, when one makes an arbitrary deformation $J \nabla_{M} \varphi$ with $\varphi \in C_{0}^{\infty}(N)$ for $N$ :

$$
\int_{M}\left\langle H, J \nabla_{M} \varphi\right\rangle=\int_{M} \varphi \operatorname{div}_{M}(J H)-\operatorname{div}_{M}(\varphi J H)=\int_{M} \varphi \operatorname{div}_{M}(J H) .
$$

The notion of Hamiltonian stationary Lagrangian submanifolds in a Kähler manifold was introduced by $\mathrm{Oh}[\mathrm{Oh}]$ as critical points of the volume functional under Hamiltonian variations (known to A. Weinstein as noted in $[\mathrm{Oh}]$ ). Chen-Morvan [CM] generalized it to the isotropic deformations.

As in Harvey-Lawson [HL], a submanifold $M$ in $\mathbb{C}^{n}$ is isotropic at $p \in M$ if

$$
J\left(T_{p} M\right) \perp T_{p} M,
$$

and it is isotropic if it is isotropic for every $p$. A submanifold $M$ being isotropic is equivalent to that the standard symplectic 2 -form on $\mathbb{R}^{2 n}$ vanishes on $M$. The dimension of an isotropic submanifold is at most $n$, the half real dimension of $\mathbb{C}^{n}$, and when it is $n$ the submanifold is Lagrangian.

Date: October 11, 2018.

The first authors is partially supported by NSERC, and the second author is partially supported by an NSF grant. 
For an immersed (n-1)-dimensional submanifold $L$ in the unit sphere $S^{2 n-1}$, let $u: L \rightarrow$ $S^{2 n-1}$ be the restriction of the coordinate functions in $\mathbb{R}^{2 n}$ to $L$. A point $u \in L$ is Legendrian if $T_{u} L$ is isotropic in $\mathbb{R}^{2 n}$ and

$$
J\left(T_{u} L\right) \perp u .
$$

$L$ is Legendrian if all the points $u$ are Legendrian. This is equivalent to that $L$ is an $(n-1)$ dimensional integral submanifold of the standard contact distribution on $S^{2 n-1}$. The cone $C(L)=\{r x: r \geq 0, x \in L\}$ is said to have link $L$. In this article, all links $L$ are assumed to be connected, and we shall use $\Sigma$ for 2 -dimensional link $L$.

The Hamiltonian stationary condition is a third order constrain on the submanifold $M$, as seen when $M$ is locally written as a graph over its tangent space at a point. The minimal submanifolds, a second order constrain on the local graphical representation of $M$, are automatically Hamiltonian stationary. We are particularly interested in the case that $M$ is a Lagrangian submanifold. The existence of (many) compact Hamiltonian stationary Lagrangian submanifolds in $\mathbb{C}^{n}$ versus the non-existence of compact minimal submanifolds makes the study of Hamiltonian stationary ones interesting. In this note, we shall not be concerned with the existence of Hamiltonian stationary ones; instead, we shall concentrate on the rigidity property, namely, when the Hamiltonian stationary ones reduce to special Lagrangians, in the case when the submanifold is a cone over a spherical link in $\mathbb{C}^{n}$.

A well-known fact about a link $L^{m} \subset S^{n}$ and the cone $C(L)$ over it is that $L$ is minimal in $S^{n}$ if and only if $C(L) \backslash\{0\}$ is minimal in $\mathbb{R}^{n+1}$. When $C(L)$ is Hamiltonian stationary and isotropic, possibly away from the cone vertex $0 \in \mathbb{R}^{2 n}$, we observe that the Hamiltonian stationary equation for $C(L)$ splits into two equations:

$$
\operatorname{div}_{L}\left(J H_{L}\right)=0
$$

i.e. the link $L$ is Hamiltonian stationary in $\mathbb{R}^{2 n}$ as well, and

$$
\left\langle J H_{L}, u\right\rangle=0,
$$

where $H_{L}$ is the mean curvature vector of $L$ in $\mathbb{R}^{2 n}$ and $u$ is the position vector of $L$. Moreover, if the link $L$ is isotropic in $\mathbb{C}^{n}$, then

$$
\operatorname{div}_{L}\left(J \bar{H}_{L}\right)=0
$$

where $\bar{H}_{L}=H_{L}-m u$ is the mean curvature vector of $L$ in $\mathbb{S}^{2 n-1}$; in fact,

$$
\operatorname{div}_{L}(J u)=\sum_{i=1}^{m}\left\langle D_{E_{i}}(J u), E_{i}\right\rangle=\sum_{i=1}^{m}\left\langle J D_{E_{i}} u, E_{i}\right\rangle=0
$$

as $D_{E_{i}} u$ is tangent to $L$, where $D$ is the derivative in $\mathbb{R}^{2 n}$ and $\left\{E_{1}, \cdots, E_{m}\right\}$ is an orthonormal local frame on $T L$.

Our observation is that the rigidity statements in $[\mathrm{CY}]$ for minimal links generalize to the Hamiltonian stationary setting.

Theorem 1.1. Let $\Sigma$ be a closed oriented immersed isotropic surface with genus $g_{\Sigma}$ in $S^{5} \subset \mathbb{C}^{3}$ such that the cone $C(\Sigma)$ is Hamiltonian stationary away from its vertex. Then

(1) if $g_{\Sigma}=0, \Sigma$ is Legendrian and minimal (in fact, totally geodesic);

(2) if $g_{\Sigma} \geq 1, \Sigma$ is either Legendrian or has exactly $2 g_{\Sigma}-2$ Legendrian points counting the multiplicity. 
It is known that the immersed minimal Legendrian sphere $\left(g_{\Sigma}=0\right)$ must be a great two sphere in $S^{5}$ (cf. [H, Theorem 2.7]). Simple isotropic tori $\left(g_{\Sigma}=1\right)$ can be constructed so that the Hamiltonian stationary cone $C(\Sigma)$ is nowhere Lagrangian. A family of Hamiltonian stationary (non-minimal) Lagrangian cones $C(\Sigma)$ with $g_{\Sigma}=1$ are presented by Iriyeh in [I]. Bryant's classification [Br, p.269] of minimal surfaces with constant curvature in spheres provides examples of flat Legendrian minimal tori as well as flat non-Legendrian isotropic minimal tori $\left(g_{\Sigma}=1\right)$ in $S^{5}$. The constructions of Haskins $[\mathrm{H}]$ and Haskins-Kapouleas [HK] show that there are infinitely many immersed (embedded if $g_{\Sigma}=1$ ) minimal Legendrian surfaces for each odd genus in $S^{5}$.

In general dimensions and co-dimensions, we have

Theorem 1.2. Let $L^{m}$ be a compact isotropic immersed oriented submanifold in the unit sphere $S^{2 n-1} \subset \mathbb{C}^{n}$ such that the cone $C\left(L^{m}\right)$ is Hamiltonian stationary away from its vertex. Suppose that the first Betti number of $L^{m}$ is 0 . Then, away from its vertex,

(1) when $m$ is the top dimension $n-1$, the cone $C\left(L^{n-1}\right)$ is Lagrangian and minimal (or equivalently $L^{n-1}$ is Legendrian and minimal);

(2) for $m<n-1$, the cone $C\left(L^{m}\right)$ is isotropic, and if the differential 1-form $\left\langle J H_{C\left(L^{m}\right)}, \cdot\right\rangle$ is closed then the mean curvature $H_{C\left(L^{m}\right)}$ of $C\left(L^{m}\right)$ vanishes on the normal subbundle $\operatorname{JTC}\left(L^{m}\right)$.

We make two remarks when the dimension $m$ of the link is two. First, Theorem 1.2 also implies Theorem 1.1(1). Second, if the first Betti number of $L^{2}$ is not zero $\left(g_{L^{2}}>0\right)$ and $L$ is isotropically immersed in $S^{2 n-1}$, with $2 n-1 \geq 5$, and $C(L)$ is Hamiltonian stationary away from its cone vertex, the same argument as in the proof of Theorem 1.1 leads to the same conclusion as in part (2) of Theorem 1.1 that the cone $C\left(L^{2}\right)$ is isotropic either everywhere or along exactly $2 g_{L^{2}}-2=-\chi\left(L^{2}\right)$ lines.

Theorem 1.2 and Theorem 1.1 (except the totally geodesic part) remain valid for nonorientable links (note that $\chi(\Sigma)=2-g_{\Sigma}$ for a compact non-orientable surface $\Sigma$ ), see Remark 2.2 and Remark 3.1. The non-orientable version of Theorem 1.2 implies that one cannot immerse a compact non-orientable $L^{n-1}$ with zero first Betti number Hamiltonian stationarily and isotropically into $S^{2 n-1} \subset \mathbb{C}^{n}$. Otherwise, the cone $C\left(L^{n-1}\right)$ would be a special Lagrangian cone, then $C\left(L^{n-1}\right)$ would be orientable, and $L^{n-1}$ would also be orientable. In particular, there exists no isotropic Hamiltonian stationary immersion of a real projective sphere $\mathbb{R} P^{2}$ into $S^{5} \subset \mathbb{C}^{3}$.

It is interesting to find out whether there exists an isotropic Hamiltonian stationary surface in $S^{5}$ with exactly $2 g_{\Sigma}-2$ Legendrian points for $g_{\Sigma}>1$.

\section{Hopf DifFerentials ANd Proof of THEOREM 1.1}

To measure how far the isotropic $\Sigma$ is away from being Legendrian, or the deviation of the corresponding cone from being Lagrangian, we project $J u$ onto the tangent space of $\Sigma$ in $\mathbb{C}^{3}$, where $J$ is the complex structure in $\mathbb{C}^{3}$. Denote the length of the projection by

$$
f=|\operatorname{Pr} J u|^{2} .
$$


To compute the length, we need some preparation. Locally, take an isothermal coordinate system $\left(t^{1}, t^{2}\right)$ on the isotropic surface

$$
u: \Sigma \rightarrow S^{5} \subset \mathbb{C}^{3} .
$$

Set the complex variable

$$
z=t^{1}+\sqrt{-1} t^{2}
$$

Then the induced metric has the local expression with the conformal factor $\varphi$

$$
g=\varphi^{2}\left[\left(d t^{1}\right)^{2}+\left(d t^{2}\right)^{2}\right]=\varphi^{2} d z d \bar{z}
$$

We project $J u$ to each of the orthonormal basis $\varphi^{-1} u_{1}, \varphi^{-1} u_{2}$ with $u_{i}=\partial u / \partial t^{i}$. Then the sum of the squares of each projection is

$$
f=\frac{\left|\left\langle J u, u_{1}\right\rangle\right|^{2}+\left|\left\langle J u, u_{2}\right\rangle\right|^{2}}{\varphi^{2}}=\frac{4\left|\left\langle J u, u_{z}\right\rangle\right|^{2}}{\varphi^{2}}
$$

where $u_{z}=\partial u / \partial z$ and $\langle\cdot, \cdot\rangle$ is the Euclidean inner product on $\mathbb{R}^{6}$, and in particular $0 \leq f \leq 1$. In fact, $f$ is the square of the norm of the symplectic form $\omega$ in $\mathbb{C}^{3}$ restricted on the cone $C(\Sigma)$ with link $\Sigma$ :

$$
\left.\left.\omega\right|_{C(\Sigma)} \wedge * \omega\right|_{C(\Sigma)}=f \cdot \text { volume form of } C(\Sigma) \text {. }
$$

The Hamiltonian stationary condition for the cone $C(\Sigma)=r u\left(t^{1}, t^{2}\right)$ is

$$
\begin{aligned}
0 & =\operatorname{div}_{C(\Sigma)}\left(J H_{C(\Sigma)}\right) \\
& =\left\langle\partial_{r}\left(J H_{C(\Sigma)}\right), \partial_{r}\right\rangle+\frac{1}{r^{2}} \operatorname{div}_{\Sigma}\left(J \frac{1}{r} H_{\Sigma}\right) \\
& =-\frac{1}{r^{2}}\langle J H, u\rangle+\frac{1}{r^{3}} \operatorname{div}_{\Sigma}\left(J H_{\Sigma}\right) .
\end{aligned}
$$

It follows that

$$
\operatorname{div}_{\Sigma}\left(J H_{\Sigma}\right)=0
$$

and

Coupled with the isotropy condition

$$
0=\langle J H, u\rangle=-\left\langle\frac{4}{\varphi^{2}} u_{z \bar{z}}, J u\right\rangle .
$$

$$
\left\langle J u_{i}, u_{j}\right\rangle=0
$$

we have the holomorphic condition

$$
\left\langle J u, u_{z}\right\rangle_{\bar{z}}=\left\langle J u_{\bar{z}}, u_{z}\right\rangle+\left\langle J u, u_{z \bar{z}}\right\rangle=\left\langle J u,-\frac{\varphi^{2}}{2} u\right\rangle=0 .
$$

The induced metric $g$ yields a compatible conformal structure on the oriented surface $\Sigma$ which makes $\Sigma$ a Riemann surface. We shall consider two cases according to the genus $g_{\Sigma}$.

(1) $g_{\Sigma}=0$ : By the uniformization theorem for Riemann surfaces (cf. [AS, p.125, p.181]), there exists a holomorphic covering map

$$
\Phi:\left(S^{2}, g_{\text {canonical }}\right) \rightarrow(\Sigma, g)
$$


or locally

$$
\Phi:\left(\mathbb{C}^{1}, \frac{1}{\left(1+|w|^{2}\right)^{2}} d w d \bar{w}\right) \rightarrow(\Sigma, g)
$$

For $z=\Phi(w)$ one has

$$
\frac{1}{\left(1+|w|^{2}\right)^{2}} d w d \bar{w}=\Phi^{*}\left(\psi^{2} g\right)=\Phi^{*}\left(\psi^{2} \varphi^{2} d z d \bar{z}\right)=\psi^{2} \varphi^{2}\left|z_{w}\right|^{2} d w d \bar{w},
$$

where $\psi$ is a positive (real analytic) function on $\Sigma$. In particular

$$
\left|z_{w}\right|^{2}=\frac{1}{\psi^{2} \varphi^{2}\left(1+|w|^{2}\right)^{2}} .
$$

Note that

$$
\left\langle J u, u_{w}\right\rangle=\left\langle J u, u_{z}\right\rangle z_{w}=\left\langle J u, u_{z}\right\rangle \frac{1}{w_{z}}
$$

is a holomorphic function of $z$, in turn it is a holomorphic function of $w$. Also $\left\langle J u, u_{w}\right\rangle$ is bounded, approaching 0 as $w$ goes to $\infty$, because

$$
\left|\left\langle J u, u_{w}\right\rangle\right|^{2}=\frac{\left|\left\langle J u, u_{z}\right\rangle\right|^{2}}{\varphi^{2}} \frac{1}{\psi^{2}\left(1+|w|^{2}\right)^{2}} .
$$

So $\left\langle J u, u_{w}\right\rangle \equiv 0$. Therefore $f \equiv 0$ and $\Sigma$ is Legendrian. We conclude that $C(\Sigma) \backslash\{0\}$ is Lagrangian.

The 1-form $\left\langle J H_{C(\Sigma)}, \cdot\right\rangle$ on the Lagrangian submanifold $C(\Sigma) \backslash\{0\}$ is closed. (This follows directly either from Theorem 3.4 of Dazord in [HL], or can be verified by locally exactness via the local expression

$$
H_{C(\Sigma)}=-J \nabla_{C(\Sigma)} \theta
$$

given in [ $[\mathrm{HL}]$; this will be done in next section.) Its restriction along $\Sigma$ is therefore a closed 1 -form $i^{*}\left\langle J H_{C(\Sigma)}, \cdot\right\rangle$, as the pullback by the inclusion $i: \Sigma \rightarrow C(\Sigma)$ of a closed 1-form. Since the first Betti number of $\Sigma$ is zero $\left(g_{\Sigma}=0\right)$, there is a smooth function $\theta_{\Sigma}$ on $\Sigma$ such that

$$
d \theta_{\Sigma}=i^{*}\left\langle J H_{C(\Sigma)}, \cdot\right\rangle \text {. }
$$

Then

$$
\left\langle\nabla_{\Sigma} \theta_{\Sigma}, \cdot\right\rangle=d \theta_{\Sigma}=\left\langle J H_{\Sigma}, \cdot\right\rangle
$$

As we have seen, the Hamiltonian stationary condition on $C(\Sigma)$ implies

$$
\begin{aligned}
0 & =\operatorname{div}_{\Sigma}\left(J H_{\Sigma}\right) \\
& =\operatorname{div}_{\Sigma}\left(\nabla_{\Sigma} \theta_{\Sigma}\right) \\
& =\Delta_{g} \theta_{\Sigma} .
\end{aligned}
$$

On the closed surface $\Sigma$, then $\theta_{\Sigma}$ is constant, in turn, $\Sigma$ is minimal.

An immersed minimal Legendrian 2 -sphere in $\mathbb{S}^{5}$ is totally geodesic. This is a known fact, for a proof, see for example in $[\mathrm{CY}]$.

(2) $g_{\Sigma} \geq 1:$ As in the $g_{\Sigma}=0$ case, the isotropic and Hamiltonian stationary condition gives us a local holomorphic function $\left\langle J u, u_{z}\right\rangle$ and global holomorphic Hopf 1-differential 
$\left\langle J u, u_{z}\right\rangle d z$. We only consider the case that $\left\langle J u, u_{z}\right\rangle d z$ is not identically 0 . The zeros of $\left\langle J u, u_{z}\right\rangle$ are therefore isolated and near each of the zeros, we can write

$$
\left\langle J u, u_{z}\right\rangle=h(z) z^{k}
$$

where $h$ is a local holomorphic function non-vanishing at the zero point $z=0$ and $k$ is a positive integer. One can also view

$$
\left\langle J u, u_{z}\right\rangle=\frac{1}{2}\left(\left\langle J u, u_{1}\right\rangle-\sqrt{-1}\left\langle J u, u_{2}\right\rangle\right)
$$

as the tangent vector

$$
\frac{1}{2}\left\langle J u, u_{1}\right\rangle u_{1}-\frac{1}{2}\left\langle J u, u_{2}\right\rangle u_{2}=\frac{1}{2}\left\langle J u, u_{1}\right\rangle \partial_{1}-\frac{1}{2}\left\langle J u, u_{2}\right\rangle \partial_{2}
$$

along the tangent space $T \Sigma$, where $\partial_{i}=\partial u / \partial t^{i}$. The projection $\operatorname{Pr} J u$ on the tangent space of $T \Sigma$ is locally represented as

$$
\operatorname{Pr} J u=\frac{\left\langle J u, u_{1}\right\rangle \partial_{1}+\left\langle J u, u_{2}\right\rangle \partial_{2}}{\varphi^{2}} .
$$

The index of the globally defined vector field $\operatorname{Pr} J u$ at each of its singular points, i.e. where $\operatorname{Pr} J u=0$, is the negative of that for the vector field $\frac{1}{2}\left\langle J u, u_{1}\right\rangle \partial_{1}-\frac{1}{2}\left\langle J u, u_{2}\right\rangle \partial_{2}$. Note that the index of the latter is $k$.

From the Poincaré-Hopf index theorem, for any vector field $V$ with isolated singularities on $\Sigma$, one has

$$
\sum_{V=0} \operatorname{index}(V)=\chi(\Sigma)=2-2 g_{\Sigma} \leq 0
$$

The zeros of $\operatorname{Pr} J u$ are just the Legendrian points on $\Sigma$. So we conclude that the number of Legendrian points is $2 g_{\Sigma}-2$ counting the multiplicity. This completes the proof of Theorem 1.1.

Remark 2.1. As mentioned in the introduction, Theorem 1.1 (except the totally geodesic part) and its generalization to higher codimensions can be extended for the non-orientable links. This can be seen as follows. The Poincaré-Hopf index theorem holds on compact non-orientable surfaces, our count of the indices of the still globally defined Pr $J u$ via local holomorphic functions is valid too, and the index of a singular point of a vector field is independent of local orientations. Moreover, this index counting argument yields an alternative proof for Theorem 1.1(1) (except the totally geodesic part) and its generalization.

\section{Harmonic forms and Proof of Theorem 1.2}

Consider an immersed isotropic Hamiltonian stationary submanifold in $S^{2 n-1}$

$$
u: L^{m} \rightarrow S^{2 n-1} \subset \mathbb{C}^{n}
$$

The isotropy condition reads for any local coordinates $\left(t^{1}, \cdots, t^{m}\right)$ on $L^{m}$

$$
\left\langle J u_{i}, u_{j}\right\rangle=0
$$

with $J$ being the complex structure of $\mathbb{C}^{n}$ and $u_{i}=\partial u / \partial t^{i}$. 
The Hamiltonian stationary condition for the cone $C(\Sigma)=r u(t)$ is

$$
\begin{aligned}
0 & =\operatorname{div}_{C(L)}\left(J H_{C(L)}\right) \\
& =\left\langle\partial_{r}\left(J H_{C(L)}\right), \partial_{r}\right\rangle+\operatorname{div}_{L}\left(J\left(\frac{1}{r} H_{L}\right)\right) \\
& =-\frac{1}{r^{2}}\left\langle J H_{L}, u\right\rangle+\frac{1}{r^{3}} \operatorname{div}_{L}\left(J H_{L}\right) .
\end{aligned}
$$

Notice that $\left\langle J H_{L}, u\right\rangle$ and $\operatorname{div}_{L}\left(J H_{L}\right)$ are independent of $r$. Therefore, the equation above splits into two equations

$$
\operatorname{div}_{L}\left(J H_{L}\right)=0
$$

and

$$
0=\left\langle J H_{L}, u\right\rangle=-\left\langle\Delta_{g} u, J u\right\rangle
$$

where $g$ is the induced metric on $L$ and $\Delta_{g}$ is the Laplace-Beltrami operator of $(L, g)$.

To measure the deviation of the corresponding cone $C\left(u\left(L^{m}\right)\right)$ from being isotropic, we project $J u$ onto the tangent space of $u\left(L^{m}\right)$ in $\mathbb{C}^{n}$. Note that the projection is the vector field along $u(L)$

$$
\operatorname{Pr} J u=\sum_{i, j=1}^{m} g^{i j}\left\langle J u, u_{i}\right\rangle u_{j}
$$

where $g_{i j}=\left\langle u_{i}, u_{j}\right\rangle, 1 \leq i, j \leq m$. The corresponding 1 -form

$$
\alpha=\sum_{i=1}^{m}\left\langle J u, u_{i}\right\rangle d t^{i}
$$

is of course globally defined on $L^{m}$. In fact it is a harmonic 1-form, because $\alpha$ is closed and co-closed as verified as follows:

$$
\begin{aligned}
d \alpha & =\sum_{i, j=1}^{m}\left\langle J u, u_{i}\right\rangle_{j} d t^{j} \wedge d t^{i} \\
& =\sum_{i, j=1}^{m}\left(\left\langle J u_{j}, u_{i}\right\rangle+\left\langle J u, u_{i j}\right\rangle\right) d t^{j} \wedge d t^{i} \\
& =\sum_{i, j=1}^{m}\left\langle J u, u_{i j}\right\rangle d t^{j} \wedge d t^{i} \\
& =0
\end{aligned}
$$


and

$$
\begin{aligned}
\delta \alpha & =(-1)^{m \cdot 1+m+1} * d * \alpha \\
& =-* d\left(\sum_{i, j=1}^{m}(-1)^{j+1} \sqrt{g} g^{i j}\left\langle J u, u_{i}\right\rangle d t^{1} \wedge \cdots \wedge \widehat{d t^{j}} \wedge \cdots \wedge d t^{m}\right) \\
& =-* \sum_{i, j=1}^{m} \partial_{j}\left(\sqrt{g} g^{i j}\left\langle J u, u_{i}\right\rangle\right) d t^{1} \wedge \cdots \wedge d t^{j} \wedge \cdots \wedge d t^{m} \\
& =-\frac{1}{\sqrt{g}} \sum_{i, j=1}^{m} \partial_{j}\left(\sqrt{g} g^{i j}\left\langle J u, u_{i}\right\rangle\right) \\
& =-\sum_{i, j=1}^{m}\left(\left\langle J u_{j}, g^{i j} u_{i}\right\rangle+\left\langle J u, \frac{1}{\sqrt{g}} \partial_{j}\left(\sqrt{g} g^{i j} u_{i}\right)\right\rangle\right) \\
& =-\left\langle J u, \Delta_{g} u\right\rangle \\
& =0,
\end{aligned}
$$

where we have used the isotropy condition and the consequence of Hamiltonian stationary condition in the last two steps, respectively.

The Hodge-de Rham theorem implies that the harmonic 1-form $\alpha$ must vanish because the first Betti number of $L^{m}$ is zero by assumption. It follows that $\operatorname{Pr} J u$ must vanish. Therefore, the cone $C\left(L^{m}\right)$ is isotropic.

Next, we claim that the differential 1-form

$$
\beta=\left\langle J H_{L}, \cdot\right\rangle
$$

on $L^{m}$ is closed. When $m=n-1$, the isotropic cone $C\left(L^{n-1}\right)$ is Lagrangian. By [HL], around each point of $C\left(L^{n-1}\right) \backslash\{0\}$, there is a locally defined Lagrangian angle $\theta$ such that

$$
H_{C(L)}=-J \nabla_{C(L)} \theta .
$$

Now the globally defined 1 -form $\beta$ on the link $L$ can be expressed locally as

$$
\beta=\left\langle\nabla_{C(L)} \theta, \cdot\right\rangle=\left\langle\nabla_{L} \theta, \cdot\right\rangle=d_{L} \theta
$$

by noticing that $H_{C(L)}=H_{L}$ as $r=1$, where the second equality holds as the two 1-forms are on $T L$ and the tangent vectors to $L$ are orthogonal to $\partial_{r}$, and $d_{L}$ stands for the exterior differentiation on $L$. We conclude that $\beta$ is a closed 1 -form on $L$. When $m<n-1$, the 1 -form $\left\langle J H_{C(L)}, \cdot\right\rangle$ is closed by assumption, so its restriction $\beta$ on $L$ is closed.

Since the first Betti number of $L$ is zero, there is a smooth function $\theta_{L}$ on $L$ such that

$$
\left\langle J H_{L}, \cdot\right\rangle=d_{L} \theta_{L} \cdot
$$

This implies that the projection of $J H_{L}$ onto $T L$ satisfies

$$
\sum_{i=1}^{m}\left\langle J H_{L}, E_{i}\right\rangle E_{i}=\nabla_{L} \theta_{L}
$$


where $\left\{E_{1}, \ldots, E_{m}\right\}$ is a local orthonomal frame of $T L$. The Hamiltonian stationary condition on $C(L)$ asserts, as we have seen earlier, that

$$
\begin{aligned}
\Delta_{L} \theta_{L} & =\operatorname{div}_{L} \nabla_{L} \theta_{L} \\
& =\sum_{i=1}^{m} \operatorname{div}_{L}\left(\left\langle J H_{L}, E_{i}\right\rangle E_{i}\right) \\
& =\sum_{i=1}^{m}\left\langle\nabla_{L}\left\langle J H_{L}, E_{i}\right\rangle, E_{i}\right\rangle+\left\langle J H_{L}, E_{i}\right\rangle \operatorname{div}_{L} E_{i} \\
& =\operatorname{div}_{L}\left(J H_{L}\right)+\left\langle J H_{L}, H_{L}\right\rangle \\
& =0 .
\end{aligned}
$$

On the closed submanifold $L$, then $\theta_{L}$ is constant. In turn, for $m=n-1, C\left(L^{n-1}\right)$ is minimal, and for $m<n-1, C\left(L^{m}\right)$ is partially minimal, namely $H_{C\left(L^{m}\right)}$ vanishes on the normal subbundle $J T C\left(L^{m}\right)$. The proof of Theorem 1.2 is complete.

Remark 3.1. As the projection $\operatorname{Pr} J u$ and the adjoint operator $\delta$ is independent of the local orientations and the Hodge-de Rham theorem holds for compact non-orientable manifolds (cf. [LW, p.125-126]), we see that Theorem 1.2 remains true for non-orientable links $L^{m}$.

Remark 3.2. For a surface link $L^{2} \subset \mathbb{S}^{2 n-1}$ with $g_{L}=0$ for the case $n>3$, if it is isotropic and $C\left(L^{2}\right)$ is Hamiltonian stationary, the same argument as in [CY] leads to the conclusion that the second fundamental form of $L$ in $\mathbb{S}^{2 n-1}$ vanishes in the normal subbundle $J u \oplus J T L$. When $n=3, L$ is totally geodesic in $\mathbb{S}^{5}$ as noted before.

Corollary 3.1. Let $L^{m}$ be a compact immersed isotropic submanifold in the unit sphere $S^{2 n-1} \subset \mathbb{C}^{n}$. If the Ricci curvature of $L^{m}$ is nonnegative, and it is positive somewhere or the Euler characteristic $\chi\left(L^{m}\right)$ is not zero, then the Hamiltonian stationary cone $C\left(L^{m}\right)$ is isotropic, in particular, $C\left(L^{n-1}\right)$ is Lagrangian (or equivalently $L^{n-1}$ is Legendrian) and minimal when $m$ is the top dimension $n-1$.

Under the above condition, from Bochner [B, p.381], it follows immediately that the first Betti number of $L^{m}$ is zero. Then Theorem 1.2 and its non-orientable version imply the corollary.

\section{REFERENCES}

[AS] L. Ahlfors and L. Sario, Riemann Surfaces, Princeton University Press, 1960.

[B] S. Bochner, Curvature and Betti numbers, Ann. Math. 49 (1948), 379-390.

[Br] R. Bryant, Minimal surfaces of constant curvature in $S^{n}$, Trans. Amer. Math. Soc. 290 (1985), no. 1, 259-271.

[CM] B.-Y. Chen, and J.-M. Morvan, Deformations of isotropic submanifolds in Kähler manifolds., J. Geom. Phys. 13 (1994), no. 1, 79-104.

[CY] J.-Y. Chen and Y. Yuan, Minimal cones with isotropic links, Int. Math. Res. Not. 2006, Art. ID 69284, $14 \mathrm{pp}$.

[D] P. Dazord, Sur la géometie des sous-fibrés et des feuilletages lagrangiense, Ann. Sci. Eć. Norm. Super., IV, Ser.13 , 465-480 (1981).

[HL] R. Harvey and H.B. Lawson, Calibrated geometries, Acta Math. 148 (1982), 47-157.

[H] M. Haskins, Special Lagrangian cones, Amer. J. Math. 126 (2004), no. 4, 845-871. 
[HK] M. Haskins and N. Kapouleas, Special Lagrangian cones with higher genus links, Invent. Math. (2007), 167, pp. 223-294.

[I] H. Iriyeh, Hamiltonian minimal Lagrangian cones in $C^{m}$, Tokyo J. Math. 28 (2005), no. 1, 91-107.

[LW] H. B. Lawson and M.-L. Michelsohn, Spin geometry, Princeton University Press, 1994.

[Oh] Y.-G. Oh, Volume minimization of lagrangian submanifolds under hamiltonian deformations, Math Z., 212 (1993), 175-192.

Department of Mathematics, University of British Columbia, Vancouver, B.C., V6T 1Z2, Canada

E-mail address: jychen@math.ubc.ca

Department of Mathematics, Box 354350, University of Washington, Seattle, WA 98195, USA

E-mail address: yuan@math. washington.edu 